\title{
A Provider - Patient Relationship: The Critical First Step of Smoking Cessation
}

\author{
Katie Jordan, OTD, OTR/L
}

Chan Division of Occupational Science and Occupational Therapy, University of Southern California, Los Angeles, CA, USA.

J Gen Intern Med 35(1):10-1

DOI: $10.1007 / \mathrm{s} 11606-019-05276-0$

(c) Society of General Internal Medicine 2019

$\mathrm{S}$ moking is the leading preventable cause of disease, disability, and death in the United States, responsible for one in every five deaths annually. ${ }^{1,2}$ In 2014, we marked the 50th anniversary of the U.S. Surgeon General's 1964 report entitled, "Smoking and Health," the first comprehensive report to link smoking to serious health consequences, including morbidity and mortality. Unlike prior releases, the authorative 1964 report significantly impacted scientific and public perspectives on health risks associated with smoking. ${ }^{2}$

At a population health level, there is much to celebrate with respect to current tobacco use in the U.S. The CDC Office on Smoking and Health (2019) reports consistent declines in adult cigarette smoking since 1965. More adults are attempting to quit, and those that continue smoking do so less frequently. Still, some stark facts dim this bright picture. There are important disparities in exposure to tobacco products based on race, ethnicity, socioeconomic status, geographic location, sexual orientation and gender identity, as well as the presence of mental health and/or substance use disorders. ${ }^{1}$ In addition, cigarette smoking continues to be associated with substantial declines in health-related quality of life and financial and societal burden, including increased medical expenditures and decreased productivity. $^{3}$

One direct consequence of the 1964 Surgeon General's report was that the National Health Interview Survey (NHIS) began tracking rates of cigarette smoking. ${ }^{1}$ In this issue of JGIM, Huo and colleagues use NHIS data to provide insights about patient-provider discussions related to smoking cessation from 2011 to 2015, a time period during which guidelines recommending screening for lung cancer among heavy smokers were first introduced. The results showed that the proportion of current smokers who reported having discussions about tobacco use with a health care provider modestly increased from $51.3 \%$ in 2011 to $55.4 \%$ in 2015 . However, many smokers - including $15 \%$ of those who underwent lung cancer screening - did not have, or could not recall, a cessation

Published online November 12, 2019 discussion with their provider. Furthermore, discussions about smoking were significantly less likely among smokers without health insurance, those who reported fewer physician visits, or those who identified as Hispanic (Huo et al. 2019).

These findings are noteworthy. A strong relationship between a care team and a patient is crucial, particularly with respect to behavior change. ${ }^{4}$ Patients who are activated in their care are more likely to be engaged in positive behavior modifications and the creation of health-promoting habits and routines. ${ }^{5}$ To achieve smoking cessation, providers must effectively connect with patients in ways that acknowledge each patient's readiness for change. ${ }^{6}$ Prior research has shown that when these principles are followed, even brief provider-patient discussions related to tobacco cessation can have meaningful effects on cessation rates. ${ }^{7}$

In their manuscript, Huo and colleagues note that survey respondents with health insurance were significantly more likely than the uninsured to receive cessation counseling, presumably due to better access to care. But access to primary care providers alone may be insufficient. Primary care clinicians must already address dozens of topics during 15-20 min encounters - leaving little time for high quality counseling. Importantly, over $60 \%$ of primary care physicians report burnout, which negatively affects practitioner well-being, workforce retention, as well as patient care and safety. ${ }^{8}$ To meet the growing challenges and demands, a team-based model that leverages the strengths of all clinicians must become the standard of care. Comprehensive tobacco use reduction efforts initiated or led by physician assistants, nurse practitioners, pharmacists, and occupational therapists have the ability to expand capacity in primary care to manage the nuanced task of cessation counseling. In fact, recent evidence indicated that nursing-led preventive/wellness visits that address smoking status are as effective in achieving smoking cessation as physician-led efforts. ${ }^{9}$

Here at the University of Southern California (USC), our team-based approach relies heavily on occupational therapists through direct one-to-one in person sessions or via telehealth. Providers can refer patients who screen positive for tobacco use to occupational therapy services through the electronic health record. USC occupational therapists utilize a specialized intervention called Lifestyle Redesign $®$ to help patients create healthy habits and routines resulting in successful behavior modification like smoking cessation. We have found that this model not only relieves the burden for cessation 
counseling from primary care clinicians, but also ensures that patients receive high-quality, evidence-based support.

The thought-provoking study by Huo et al. highlights that although we have made substantial progress with respect to tobacco cessation, much work remains. In particular, we must better leverage clinical team members who can effectively bring about tobacco use reduction. Providers and consumers must also advocate to ensure adequate resources for team-based approaches to counseling. Through these efforts, we have a particular obligation to address the alarming disparities in rates of tobacco use.

This study makes an important contribution to our understanding of modern tobacco counseling practices, highlighting the need for new approaches to ensure that all patients have access to effective, accessible, sustainable, and equitable solutions for smoking cessation.

Corresponding Author: Katie Jordan, OTD, OTR/L; Chan Division of Occupational Science and Occupational Therapy University of Southern California, Los Angeles, CA, USA (e-mail: mkjordan@med. usc.edu).

\section{Compliance with Ethical Standards:}

Conflict of Interest: The author has no conflicts of interest to declare.

\section{REFERENCES}

1. Centers for Disease Control and Prevention. Smoking \& Tobacco Use. Available at: https://www.cdc.gov/tobacco/about/osh/index.htm. Accessed May 31, 2019.

2. U.S. Department of Health and Human Services. The health consequences of smoking-50 years of progress: a report of the surgeon general. Atlanta, GA: U.S. Department of Health and Human Services, Centers for Disease Control and Prevention, National Center for Chronic Disease Prevention and Health Promotion, Office on Smoking and Health, 2014. Available at: https://www.surgeongeneral.gov/library/reports/50-yearsof-progress/full-report.pdf. Accessed May 30, 2019.

3. Baker CL, Bruno M, Emir B, Li VW, Goren A. Smoking cessation is associated with lower indirect costs. J Occup Environ Med; 2018, 60(6), 490-495.

4. Van Liew JR, Cvengros JA, Christensen AJ. Patient-provider interactions: promoting effective behavior and providing optimal care. In: Fisher E. et al. (eds) Principles and concepts of behavioral medicine. Springer, New York, NY; 2018.

5. Greene J, Hibbard JH. Why does patient activation matter? An examination of the relationships between patient activation and health-related outcomes. J General Internal Med; 2012, 27(5), 520-526.

6. Luck $\mathbf{K}$, Beagan B. Occupational transition of smoking cessation in women: "You're restructuring your whole life." J Occup Sci; 2014, 22(2), 183-196.

7. World Health Organization. WHO framework convention on tobacco control; 2005. Retrieved from http://www.who.int/fctc/text_download/ en/. Accessed May 30, 2019.

8. Willard-Grace R, Knox M, Huang B, Hammer H, Kivlahan C, Grumbach K. Burnout and health care workforce turnover. Ann Family Med; 2019, $17(1), 36-41$.

9. Bryers MA, Wright P, Tilford JM, Nemeth LS, Matthews E, Mitchell A. Comparing smoking cessation outcomes in nurse-led and physician-led primary care visits. J Nursing Care Quart; 2018, 33 (3), 272-278.

Publisher's Note Springer Nature remains neutral with regard to jurisdictional claims in published maps and institutional affiliations. 